\title{
Statistical properties of volume and calendar effects in prediction markets
}

\author{
Valerio Restocchi ${ }^{\mathrm{a}, \mathrm{b}}$, Frank McGroarty ${ }^{\mathrm{a}}$, Enrico Gerding ${ }^{\mathrm{b}}$ \\ ${ }^{a}$ Southampton Business School, University of Southampton, SO17 1BJ, Southampton, UK \\ ${ }^{b}$ Electronics and Computer Science, University of Southampton, SO17 1BJ, Southampton, \\ $U K$
}

\begin{abstract}
Prediction markets have proven to be an exceptional tool for harnessing the "wisdom of the crowd", consequently making accurate forecasts about future events. Motivated by the lack of quantitative means of validations for models of prediction markets, in this paper we analyze the statistical properties of volume as well as the seasonal regularities (i.e., calendar effects) shown by volume and price. To accomplish this, we use a set of 3385 prediction market time series provided by PredictIt. We find that volume, with the exception of its seasonal regularities, possesses different properties than what is observed in financial markets. Moreover, price does not seem to exhibit any calendar effect. These findings suggest a significant difference between prediction and financial markets, and offer evidence for the need of studying prediction markets in more detail.
\end{abstract}

Keywords: Prediction markets; Political markets; Stylized facts; Long memory; Power-law behavior.

\section{Introduction}

Prediction markets are effective tools that harness the wisdom of the crowd to make accurate forecasts on a number of events (Berg, Nelson, and Rietz, 2008). Although prediction markets are most famous for allowing anyone to bet on political events, often resulting in better predictions on political election outcomes than polls and experts (Wolfers and Zitzewitz, 2006), they are also used in many other contexts, e.g., to forecast business output by companies such as Google, Intel, and General Electrics, to predict the likelihood of natural disasters, or the future value of macroeconomic parameters (Plott and Chen, 2002; Cowgill, Wolfers, and Zitzewitz, 2009). Moreover, due to features such as possessing a definite end-point, prediction markets represent an ideal test bed

\footnotetext{
* Corresponding author

Email addresses: vr2g14@soton.ac.uk (Valerio Restocchi), f.j.mcgroarty@soton.ac.uk (Frank McGroarty), eg@ecs.soton.ac.uk (Enrico Gerding)
} 
to study decision making under uncertainty. This allows, opposite to financial markets, to observe the outcome of an event, and all uncertainty is resolved at a fixed point-in-time.

However, historical insufficiency of data has limited the number of empirical studies of prediction markets. Notably, there is no comprehensive work on the empirical regularities observed in prediction markets (or stylized facts), whereas in financial markets data-driven analysis has always represented a prominent, valuable field of study (Mantegna and Stanley, 2000; Cont, 2001). One of the main consequences is that quantitative models of prediction markets lack an important means of validation.

In this paper, we focus on the analysis of daily volumes (measured as the number of shares traded on a given day), and calendar effects, i.e., regularities that occur during a trading period, such as a week, or a year. We find that volume in political prediction markets shares only few of the characteristics typical of stock market time series. Specifically, we find that volume properties, including calendar effects, seem to be similar to those observed in the stock market, whereas we find no evidence of any price seasonalities.

The paper is organized as follows. In Section 2 we present the data set and explain how prediction markets work. In Section 3, we perform a statistical analysis of volume, and Section 4 depicts our findings on volume and price calendar effects. Finally, in Section 5 we summarize and discuss our results.

\section{Data and Methods}

Our data set comprises the daily volumes and the OHLC contract prices of 3385 betting markets on political events, provided by PredictIt ${ }^{1}$, for a total of 112761 valid observations (i.e., after removing all days in which there was no trading activity). Contracts on the PredictIt exchange market are ArrowDebreu securities, i.e., contracts which are priced between 0 and 1 dollars, and whose payoff is either 0 or 1 dollars and solely depends on the the outcome of a future event. For instance, one could buy a contract on either "Trump will lead" or "Clinton will lead" in the market "Who will lead in Trump vs. Clinton polling on September 14?" (or sell a contract on "Clinton will lead" or "Trump will lead", respectively). Then, one contract "Clinton will lead" pays 1 dollar if Clinton will be leading in Trump vs. Clinton polling on September 14, and 0 otherwise. As a consequence, rational traders are willing to buy a contract on a given outcome only if the current price of such a contract is lower than the probability they attach to the respective outcome to occur.

To perform our analysis, we use this data in two ways. To examine the distribution of daily traded shares, we aggregate volumes across all markets, which allows us to have sufficient observations to reconstruct a significant distribution. Conversely, to examine other properties such as calendar effects, we analyze each market separately and then take both the average and the median

\footnotetext{
${ }^{1}$ www.predictit.org
} 
Table 1: Summary statistics for the distribution of traded volume.

\begin{tabular}{cccccccc}
\hline N.Observations & Mean & St.Dev. & Minimum & $q_{25 \%}$ & $q_{50 \%}$ & $q_{75 \%}$ & Maximum \\
\hline 112761 & 3515.68 & 18950.04 & 1 & 43 & 306 & 1761 & 1388889
\end{tabular}

results among all markets, which allows us to have a more detailed statistical description of these phenomena.

In the next sections, we present our findings and describe in more detail how the results are obtained.

\section{Statistical analysis of traded volume}

In this section, we analyze the statistical properties of volume, which is measured as the number of daily traded shares, from the PredictIt data set. Specifically, we examine its distribution, its temporal evolution, and its longterm memory.

\subsection{Volume distribution}

To analyze the distribution of the number of contracts traded each day for each market, we exclude those days in which no contract has been traded, which leaves 3363 markets and a total of 112761 observations (i.e., trading days with positive volume). The summary statistics of the distribution of volumes (shown in Table 1) indicate that most of the markets examined display a small number of daily trades. Specifically, we find that only in half of the days with trading activity the number of transactions is greater than 306, and only during $25 \%$ of the active days 1761 or more contracts are purchased. Also, we observe that the mean is one order of magnitude larger than the median, and the kurtosis and skewness values are high. This may indicate that the distribution of volumes is characterized by heavy tails, i.e., most of the trading activity is concentrated in few trading days. Many probability distributions that characterize natural and social phenomena display such heavy tails. More specifically, most of these distribution have a power-law like asymptotical behavior Newman (2004); Sornette (2006). In financial markets, the tails of distribution of price changes have been shown to be heavy for most stocks and indexes (Campbell, Lo, and MacKinlay, 1997; Cont, Potters, and Bouchaud, 1997) and, although the exact asymptotic behavior of such tails is still under debate (Malevergne, Pisarenko, and Sornette, 2005), the power-law decay, given by:

$$
p(x) \sim x^{-\alpha}
$$

is the most widely used (Gopikrishnan et al., 2000; Plerou et al., 2004) to fit the decay of the tails.

Both the summary statistics and Fig. 3.1 suggest that this might also be the case of our distribution. We check this by fitting the tail of our distribution by following a procedure which enables us to estimate the power-law exponent for discrete data (Bauke, 2007), and relies on a maximum likelihood estimation. 


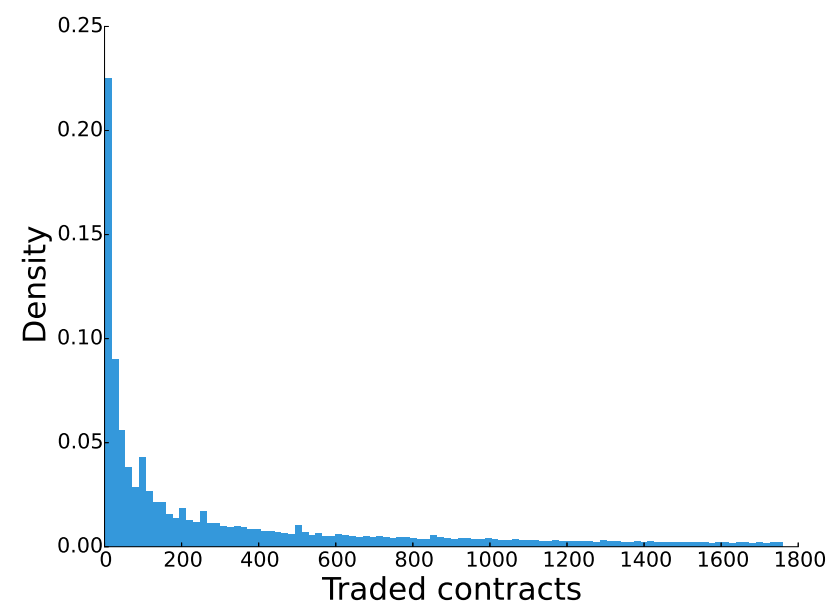

Figure 1: Distribution of the number of daily traded contracts. The distribution is shown only for $v<1761$, corresponding to the $75 \%$ of the observations.

This procedure, in contrast to other methods such as graphical methods and linear regression, is found to be more robust and reliable (Bauke, 2007; Deluca and Corral, 2013)).

In more detail, this method, which is essential to fit a PDF to a discrete power-law form (Bauke, 2007; Clauset, Shalizi, and Newman, 2009), consists in finding the value $\alpha$, such that:

$$
p(x)=\frac{x^{-\alpha}}{\Delta \zeta}
$$

where $x$ represents the daily volumes, and $\Delta \zeta$ is the difference:

$$
\Delta \zeta \equiv \zeta\left(\alpha, x_{\min }\right)-\zeta\left(\alpha, x_{\max }\right)
$$

where $\zeta$ is the Hurwitz zeta function, defined as:

$$
\zeta\left(\alpha, x_{\text {min }}\right)=\sum_{i=0}^{\infty} \frac{1}{\left(i+x_{\text {min }}\right)^{\alpha}}
$$

Here, $x_{\min }$ is the number of traded shares after which the distribution of volume starts behaving like a power law. The theoretical limit of the distribution, i.e., the largest possible value of $x$, is denoted by $x_{\max }$. However, for volumes, there is no such a constraint. Indeed, in theory, any number of shares can be exchanged during a single trading day. Therefore, we can assume that $x_{\max }=\infty$ and, consequently, $\zeta\left(\alpha, x_{\max }\right)=0$. 
Given this, it is possible to compute the likelihood function for $p(x)$, which is given by

$$
\left.L(\alpha)=-\alpha\left(\sum_{i=0}^{N} \ln \left(x_{i}\right)\right)-N \ln (\Delta \zeta)\right)
$$

Then, the maximum likelihood estimator, $\hat{\alpha}$ is given by:

$$
\hat{\alpha}=\underset{\alpha}{\operatorname{argmax}}[L(\alpha)]
$$

Since, in this case, there exists no closed-form solution for $\hat{\alpha}$, we find the value that maximizes Eq. (5) numerically.

Finally, the last step required in order to accurately estimate $\alpha$, is to find the numerical value of $x_{\min }$. To achieve this, we perform a two-sample KolgomorovSmirnov test (KS), as suggested by Clauset et al. (Clauset, Shalizi, and Newman, 2009). The procedure they introduce is as follows: first, we fix the value of $x_{m i n}$, starting from the smallest possible, and remove from our data all values of $x$ such that $x<x_{\text {min }}$, if any. Second, we fit a power-law distribution to these values, and find $\hat{\alpha}$. Third, we perform the KS test between our data and a sample drawn from a power law distribution with exponent $\hat{\alpha}$, hence computing the KS statistic (D). Finally, we increase by the smallest possible increment the value of $x_{\min }$, and we repeat the procedure until all possible values of $x_{\min }$ have been considered.

Then, we choose the $x_{\min }$ that minimizes the value of $\mathrm{D}$, and take the corresponding $\hat{\alpha}$ as the power-law exponent for our distribution. By following

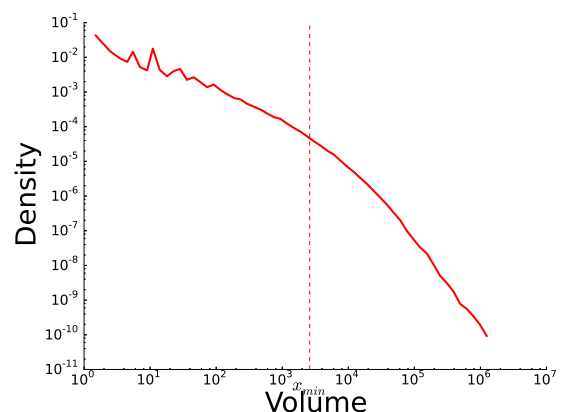

(a)

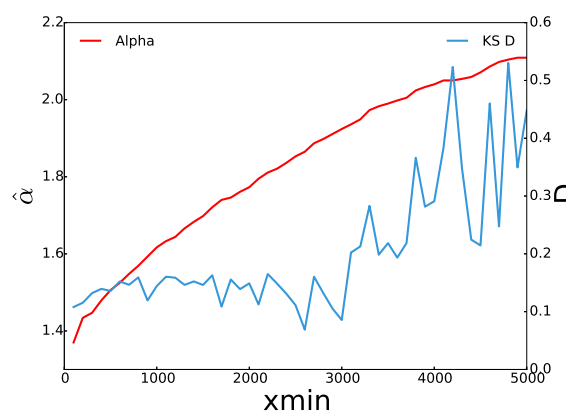

(b)

Figure 2: Figure (a) displays the PDF of volumes in a logarithmic scale. Figure (b) shows the KS statistic and the corresponding values of $\hat{\alpha}$ for different $x_{\min }$

this procedure, we find that the distribution of traded shares follows a power-law with exponent $\hat{\alpha}=1.865 \pm 0.002$ for values greater than 2600 , corresponding to the $20 \%$ of the total observations. This value is not distant from the powerlaw exponent $\gamma_{q}=1.53 \pm 0.07$ estimated for financial markets (Gopikrishnan et al., 2000; Gabaix et al., 2007), from which we can conclude that, although 
in prediction markets volumes are lower than in the stock market, the decay of the number of traded shares is similar.

\subsection{Autocorrelation of volumes}

Next, we examine the long-memory properties of volumes. To achieve this, compute the autocorrelation function of the number of traded shares, and fit it to a power-law distribution. To obtain an accurate estimation, we computed the autocorrelation function for lags in the range $1<\tau<100$, i.e., we used all markets longer than 100 days, for a total of 236 markets. We find that the volume autocorrelation function can be described as:

$$
\langle V(t), V(t+\tau)\rangle \sim \tau^{-\lambda}
$$

where we estimate the exponent to be $\lambda=0.094 \pm 0.003$ (see Fig. 3). This result

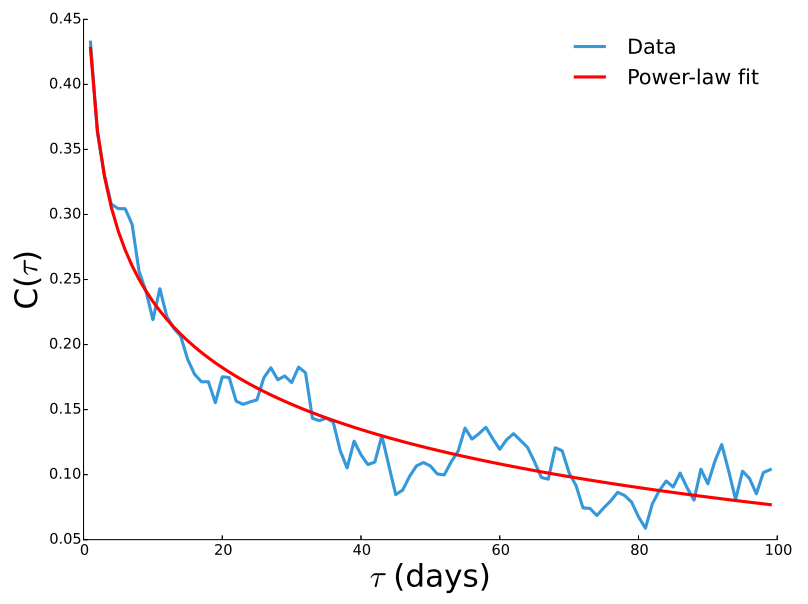

Figure 3: Autocorrelation function of traded volume and the fitted power law with exponent $\lambda=0.094$.

suggests that trading activity behaves in the same way in both prediction and stock markets, in which the power-law exponent is observed to be of the same order of magnitude. More specifically, its value is estimated to be $\lambda=0.30$ for US stocks (Plerou et al., 2001), and $\lambda=0.21$ for the Chinese stock market (Qiu et al., 2009), which also suggests that the decay of the volume autocorrelation function is faster the more liquid the market is.

\subsection{Temporal evolution of traded volume}

An interesting aspect of prediction markets time series (and, more generally, state-contingent claims) is that, in contrast to those of the stock market, they have a fixed end-point. In this section, we examine this aspect of prediction 


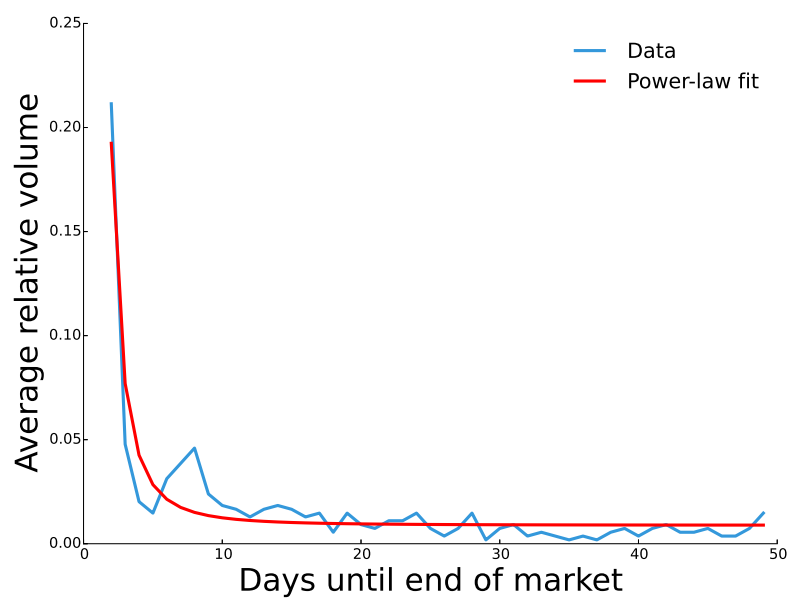

Figure 4: Relative volume depending on the number of days $\tau$ until the end of the market.

markets, i.e., the temporal distribution of volume, and find that, towards the end of the market, the average daily volume grows significantly. Specifically, the number of traded shares depends on the number of remaining days $\tau$ until the end of the market, and, as shown in Fig. 3.3, this relation follows power-law decay:

$$
V(T-\tau) \sim \tau^{-\zeta}
$$

where $\mathrm{T}$ denotes the final day of the market. We fit this function with a power law, and we estimate the exponent to be $\zeta=2.44 \pm 0.06$, which suggests that the during the last days of trading, volumes are higher than during all the rest of trading days combined. This result can be explained in several ways. For example, those who invest in prediction markets, may be waiting for a lower uncertainty on the outcome (i.e., waiting for new information to be revealed), or they simply have a higher utility to bet in the days right before the end of the market, hence reducing the time between the investment and the (potential) gain. Either way, we believe this is a crucial result for building realistic models of prediction markets, because this phenomenon may generate non-trivial price dynamics during the last days of trading.

\subsection{Volume-volatility correlation}

In this section we examine the correlation between volume and price in prediction markets. In the stock market, it has been observed in a number of contexts that volume changes and the volatility of returns are correlated (Chordia, Roll, and Subrahmanyam, 2001; Podobnik et al., 2009). For instance, it is shown that volatility grows proportionally to the total number of trades in a market (Podobnik et al., 2009). Unfortunately, for the prediction markets, we do 
not possess order-level data, and hence we show that volume and volatility are correlated on a daily time scale. That is, we compute the correlation coefficient
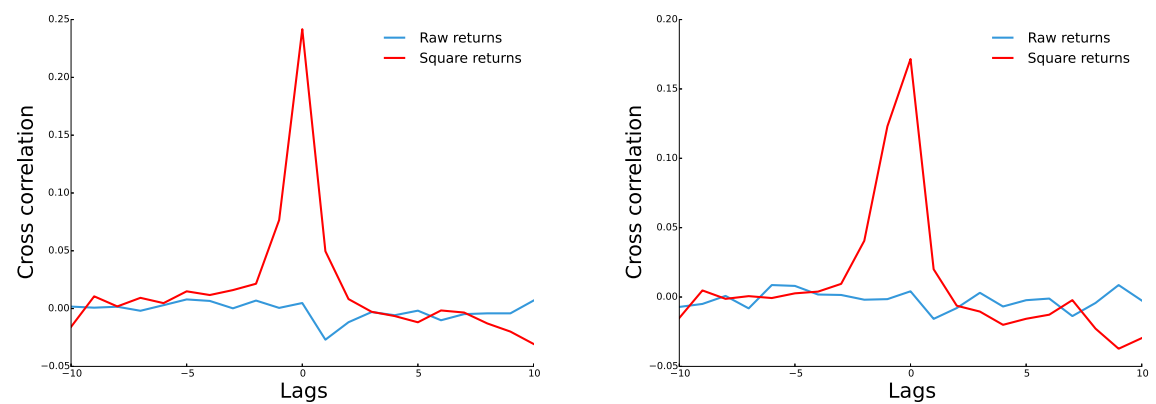

Figure 5: Figure (a) shows the cross-correlation between traded volume and both volatility (square returns) and price changes (raw returns). Figure (b) shows the cross-correlation between changes in traded volume and both volatility (square returns) and price changes (raw returns)

$$
C(\tau)^{s q}=\left\langle r_{t}^{2}, v(t+\tau)\right\rangle
$$

and find that correlation is significant only for $\tau=0$. Fig. 3.4 shows the cross correlation function between traded volume and volatility and also between traded volume and raw returns, defined as:

$$
C(\tau)=\left\langle r_{t}, v(t+\tau)\right\rangle
$$

for which the correlation coefficient is insignificant at all lags $\tau$. This implies that volume is only correlated with volatility (at lag 0 ) but not with price changes, which is a well known fact in financial markets (Podobnik et al., 2009). Interestingly, we find similar results when computing the cross correlation between returns and volume changes (Fig. 3.4). This is in contrast to what is found in the stock market, for which it has been observed that the correlation between volume changes and volatility decays with a power law (Podobnik et al., 2009). Conversely, in our data set we find that volatility is correlated with volume changes only at lag 0 .

\section{Calendar Effects}

Calendar effects, or seasonalities, are cyclical regularities that occur throughout a trading period, be it a year, a week, or a day, and have been observed in both returns and volume by a number of authors who examined international stock markets (Sewell, 2011). In this section we examine some well-known effects that are present in financial markets (Dzhabarov and Ziemba, 2010), and we find that only some of them can be observed in prediction markets. Specifically, we first describe cyclical regularities exhibited by trading activity and then focus 
on price changes, for which we examine the Weekend and the January effects in detail.

\subsection{Trading activity calendar effects}

There is evidence that, in financial markets, trading activity significantly varies depending on the time of the day and the day of the week. The first comprehensive study of volume calendar effects (Jain and Joh, 1988) examines several years of NYSE-listed stock data and find that liquidity is lowest on Monday, peaks on Wednesday, and drops until Friday. A similar, more recent study (Chordia, Roll, and Subrahmanyam, 2001), which analyzes U.S. stocks between 1988 and 1998, find that the volume peak has shifted to Tuesdays, whereas Fridays have become the days with the lowest liquidity. In this section

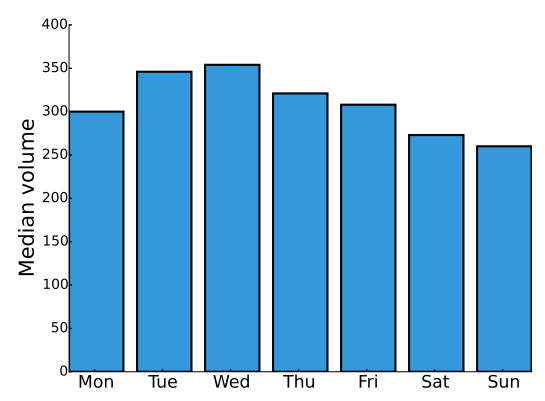

(a)

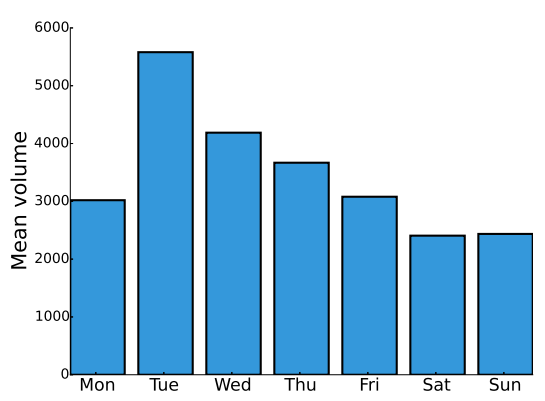

(b)

Figure 6: Figure (a) and Figure (b) display the median and the mean, respectively, number of contracts traded by day of the week.

we analyze trading activity in our data, and find that it significantly varies across days of the week and across months of the year. Although this behavior is similar to that of the U.S. stock market, this is a non-trivial result, since prediction markets possess two main differences compared with stock markets. Specifically, in prediction markets, it is possible to trade during weekends. Also, since liquidity in prediction markets is much lower than in financial markets, we find that the average number of traded shares is significantly affected by those markets in which volumes are largest. Specifically, to overcome this issue, we present our results using both the average and the median volumes.

Despite these differences, we find that most of our results are comparable with those of U.S. stocks. In fact, we conclude that, in our data, trading activity is lowest during weekends, but otherwise shows a trend similar to that found in the U.S. stock market (see Fig. 6). Table 2 shows that the average volume is low on Mondays, peaks on Tuesdays, and then decreases gradually for the rest of the week, and it reaches its lowest value during weekends, which agrees with the analysis by Chordia, Roll, and Subrahmanyam (2001). The analysis of the median number of traded shares (Fig. 6, and Table 2) shows a similar 
Table 2: This table displays summary statistics of the trading activity (expressed as the number of contracts traded) across the days of the week. The t statistic is used to either accept or reject the null hypothesis that the mean volume value of a given day of the week is the same as the mean value for the other days.

\begin{tabular}{|c|c|c|c|c|c|c|c|}
\hline & Monday & Tuesday & Wednesday & Thursday & Friday & Saturday & Sunday \\
\hline Mean & 3019.45 & 5580.16 & 4187.65 & 3667.96 & 3079.47 & 2406.12 & 2436.10 \\
\hline St. Dev. & 10550.43 & 36248.83 & 18319.36 & 19204.85 & 11540.58 & 10730.70 & 8266.70 \\
\hline Median & 300 & 346 & 354 & 321 & 308 & 273 & 260 \\
\hline t-stat. & $-5.50^{*}$ & $8.55^{*}$ & $5.09^{*}$ & $1.11^{* *}$ & $-4.62^{*}$ & $-11.85^{*}$ & $-13.34^{*}$ \\
\hline
\end{tabular}

pattern, although the volume differences across the days of the week become less pronounced compared to the average value, and the number of traded contracts has a high on Wednesdays instead. We repeat the analysis for the months of the year, and we find that, although the differences between mean and median are more pronounced than in the weekly analysis, both measures show similar trends (see Fig. 7). First, January and December are the months with the least trades in both cases. Second, both the mean and the median volumes increase from January to Spring (April and March for the median and the mean value, respectively), then have a local low in August, and then a new high in Autumn (October for the median volume, November for the mean volume). These findings suggest that, despite the structural differences, volume temporal regularities in prediction markets are similar to those found in stock markets.

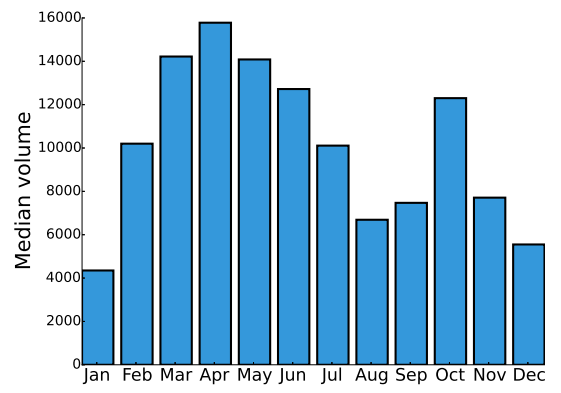

(a)

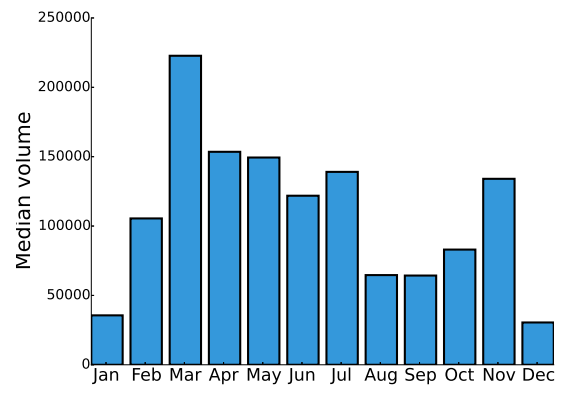

(b)

Figure 7: Figure (a) and Figure (b) display the median and the mean number of contracts traded by month of the year, respectively.

\subsection{Price calendar effects}

In this section, we examine price changes across days of the week and months of the year. We first introduce these regularities, also presenting the results found in financial markets, and then show that these two patterns are not exhibited by our data. Indeed, we find that, opposite to volume, price in prediction 
Table 3: This table displays summary statistics of the trading activity (expressed as the number of contracts traded) across the months of the year. The t statistic is used to either accept or reject the null hypothesis that the mean volume value of a given day of the week is the same as the mean value for the other days.

\begin{tabular}{|c|c|c|c|c|c|c|c|c|c|c|c|c|}
\hline & Jan & Feb & Mar & Apr & May & Jun & Jul & Aug & Sep & Oct & Nov & Dec \\
\hline Mean & 35621.59 & 105487.06 & 222737.46 & 153514.76 & 149391.7 & 121854.35 & 139041.75 & 64646.92 & 64314.74 & 83033.61 & 134040.53 & 30493.27 \\
\hline St. Dev. & 18903.99 & 66802.39 & 156852.56 & 140245.76 & 183499.35 & 112634.55 & 118178.35 & 42586.11 & 45412.31 & 49532.94 & 151787.61 & 13093.59 \\
\hline Median & 4350.0 & 10200.0 & 14220.0 & 15780.0 & 14085.0 & 12720.0 & 10110.0 & 6690.0 & 7470.0 & 12300.0 & 7710.0 & 5550.0 \\
\hline t-stat. & $-35^{*}$ & $0.02^{* * *}$ & $12.39^{*}$ & $5.83^{*}$ & $4.46^{*}$ & $2.79^{*}$ & $5.65^{*}$ & -15.35 * & $-15.37^{*}$ & $-8.63^{*}$ & $3.2^{*}$ & -40.36 \\
\hline
\end{tabular}

markets does not follow the same behavior as in the stock market and, more generally, does not seem to exhibit any regularity. Conversely, in numerous stock markets, it has been observed that prices display more calendar regularities than volume, and the study of this topic has generated a large body of literature (Thaler, 1992; Constantinides, Harris, and Stulz, 2003). After their discovery, many of these anomalies have reduced or even disappeared (Mclean and Pontiff, 2016), but some of the most important calendar effects, among which the January effect and the Weekend effect are the most documented (Sewell, 2011), are still present in many stock markets (Dzhabarov and Ziemba, 2010).

\subsection{The Weekend and the January effects}

The weekend effect (sometimes referred to as Monday effect) is an empirical regularity by which average returns on Mondays are significantly lower than those of the rest of the week, and is often regarded as the strongest of calendar effects (Rubinstein, 2001). This anomaly was firstly observed in the 1930s (Fields, 1931), but the first comprehensive discussion was provided by Kenneth French (French, 1980), who analyzed more than twenty years of stock returns in the U.S. market to test two hypotheses. The first, called calendar time hypothesis, states that the expected returns on Mondays should be three times those for the other days of the week, since the the risk accumulated during weekends should be reflected in Monday's returns. The second, named trading time hypothesis, states that, if only trading time matters to generate returns, there should be no distinction between Mondays and other days. However, French found that neither of these hypotheses were true. In fact, he found that, on average, Mondays display lower returns than all of other days of the week and, more specifically, Monday is the only day of the week during which average returns are negative.

Lakonishok and Maberly (Lakonishok and Maberly, 1990) provide an explanation of the weekend effect based on the analysis of trading patterns of individual and institutional investors. First, they find that, on Mondays individual investors tend to trade more compared with the rest of the week, and also that the number of sell transactions relative to buy transactions increase significantly. Second, they observe that, in their data, the traded volume by institutional investors was the lowest on Mondays. They claim that these two regularities combined provide a partial explanation for the weekend effect. 


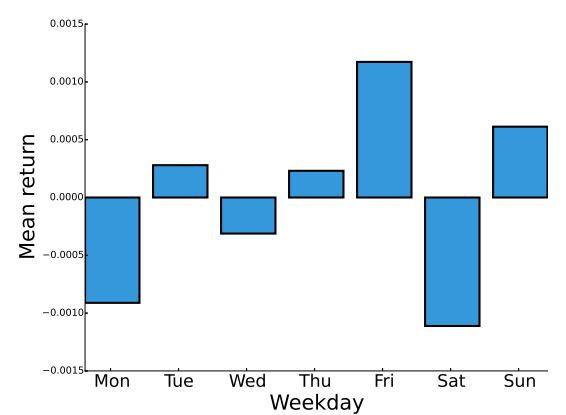

(a)

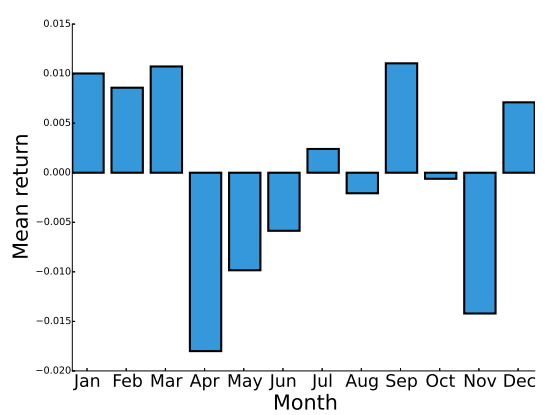

(b)

Figure 8: Figure (a) and Figure (b) display the mean return across days of the week and months of the year, respectively.

The January effect is another important calendar regularity, whereby returns on January are significantly higher than in other months. It has been first observed in the U.S. and Australia stock markets (Wachtel, 1942; Praetz, 1972; Officer, 1975; Rozeff and Kinney, 1976), and in several international stock markets afterwards (Gultekin and Gultekin, 1983; Agrawal and Tandon, 1994). Similarly to the weekend effect, the January effect has proven to be a regularity whose causes are puzzling (Haugen and Lakonishok, 1988). There are many competing explanation attempts, but most of these theories revolve around small firms. Indeed, there is evidence that this phenomenon is related with the capitalization of firms, and then that it is likely to be a consequence of a small-firm effect (Reinganum, 1983), low share prices (Bhardwaj and Brooks, 1992), or tax-motivated trading (Sias and Starks, 1997; Poterba and Weisbenner, 2001).

\subsection{Analysis of returns}

In this section we examine the seasonality of returns, to find whether the Weekend and the January effects exist in prediction markets. To achieve this, we follow the same procedure employed to analyze calendar effects on volume, and take into account both the mean and the median return. However, in contrast to traded volume, returns do not seem to possess any significant differences across days of the week (see Fig. 8). Mean daily returns, as it is shown in Table 4, lie between -0.001 and 0.001 for all days of the week, i.e., they are one order of magnitude smaller than the minimum possible raw return $\left|r_{t}\right|=0.01$, and these small differences disappear completely when considering the median returns. Accordingly, we find that all the p-values from the t-test are greater than 0.7, and hence the null hypothesis that average returns are the same across the days of the week cannot be rejected. Similarly, we find that monthly returns do not display any significant difference (see Table 5). These findings are consistent with the hypothesis that the January effect is due to smaller-capitalization stocks and tax-loss selling (Roll, 1983). Indeed, in prediction markets, there is no equivalent 
Table 4: This table displays summary statistics of the returns for each day of the week. The t statistic is used to either accept or reject the null hypothesis that the mean return of a given day of the week is the same as the mean return for the other days.

\begin{tabular}{cccccccc}
\hline & Monday & Tuesday & Wednesday & Thursday & Friday & Saturday & Sunday \\
\hline Mean & -0.0009 & 0.0003 & -0.0003 & 0.0002 & 0.0012 & -0.0011 & 0.0006 \\
St. Dev. & 0.11 & 0.11 & 0.11 & 0.11 & 0.14 & 0.08 & 0.07 \\
Median & 0 & 0 & 0 & 0 & 0 & 0 & 0 \\
t-stat. & $-1.11^{*}$ & $0.34^{*}$ & $-0.41^{*}$ & $0.3^{*}$ & $1.21^{*}$ & $-1.75^{*}$ & $1.1^{*}$ \\
\hline \multicolumn{7}{c}{ indicates that the result is not significant. }
\end{tabular}

Table 5: This table displays summary statistics of the returns for each month of the year. The $t$ statistic is used to either accept or reject the null hypothesis that the mean return of a given month of the year is the same as the mean return for the other months.

\begin{tabular}{ccccccccccccc}
\hline & Jan & Feb & Mar & Apr & May & Jun & Jul & Aug & Sep & Oct & Nov & Dec \\
\hline Mean & 0.01 & 0.0086 & 0.0107 & -0.018 & -0.0098 & -0.0059 & 0.0024 & -0.0021 & 0.011 & -0.0006 & -0.0142 & 0.0071 \\
St. Dev. & 0.498 & 0.579 & 0.699 & 0.545 & 0.563 & 0.579 & 0.593 & 0.614 & 0.558 & 0.582 & 0.655 & 0.549 \\
Median & 0 & 0 & 0 & 0 & 0 & 0 & 0 & 0 & 0 & 0 & 0 & 0 \\
t-stat. & $0.34^{*}$ & $0.25^{*}$ & $0.25^{*}$ & $-0.55^{*}$ & $-0.32^{*}$ & $-0.2^{*}$ & $0.08^{*}$ & $-0.07^{*}$ & $0.4^{*}$ & $-0.03^{*}$ & $-0.37^{*}$ & $0.21^{*}$ \\
\hline
\end{tabular}

indicates that the result is not significant.

of capitalization since contract prices purely reflect the likelihood of a given event to occur as perceived by market participants. Also, losses from these markets do not impact on fiscal contribution, since prediction markets fall under the gambling legislation in most countries and, importantly, volumes are too low to affect fiscal contribution whatsoever.

\section{Conclusions}

We analyzed calendar effects and several statistical properties of volumes in prediction markets, by using a data set comprising 3385 time series of security prices and trading volumes on political events. We find volume properties and price seasonalities mostly differ from those observed in financial markets, with the exception of volume calendar effects. We argue that this may be caused by the low liquidity and short duration that characterize prediction markets, especially compared with those of the stock market. These results suggest that studying prediction markets could provide additional insights on people's individual and collective behavior when investing under uncertainty, and we advocate the use of our results to build and validate new models of prediction markets.

\section{Acknowledgments}

We would like to thank all participants at the 2nd Workshop of the Econophysics Network and seminar attendees at the London Institute of Mathematical Sciences for precious comments. This research did not receive any specific grant from funding agencies in the public, commercial, or not-for-profit sectors. 


\section{References}

Agrawal, Anup and Kishore Tandon. 1994. "Anomalies or illusions? Evidence from stock markets in eighteen countries." Journal of International Money and Finance 13:083-106.

Bauke, Heiko. 2007. "Parameter estimation for power-law distributions by maximum likelihood methods." The European Physical Journal B 58 (2):167-173.

Berg, JE, FD Nelson, and TA Rietz. 2008. "Prediction market accuracy in the long run." International Journal of Forecasting 24 (285-300).

Bhardwaj, R.K. and L.D. Brooks. 1992. "The January Anomaly: Effects of Low Share Price, Transaction Costs, and Bid-Ask Bias." The Journal of Finance 47 (2):553-575.

Campbell, JY, AWC Lo, and AC MacKinlay. 1997. The econometrics of financial markets. Princeton, New Jersey: Princeton University Press.

Chordia, Tarun, Richard Roll, and Avanidhar Subrahmanyam. 2001. "Market Liquidity and Trading Activity." The Journal of Finance LVI (2).

Clauset, Aaron, CR Shalizi, and MEJ Newman. 2009. "Power-law distributions in empirical data." SIAM review 51 (4):661-703.

Constantinides, G.M., M. Harris, and R.M. Stulz. 2003. Handbook of the Economics of Finance. Amsterdam: Elsevier North-Holland.

Cont, Rama. 2001. "Empirical properties of asset returns: stylized facts and statistical issues." Quantitative Finance 1 (2):223-236.

Cont, Rama, Marc Potters, and Jean-Philippe Bouchaud. 1997. "Scaling in stock market data: stable laws and beyond." In Scale invariance and beyond. $75-85$.

Cowgill, Bo, Justin Wolfers, and E Zitzewitz. 2009. "Using prediction markets to track information flows evidence from google." In Auctions, Market Mechanisms and Their Applications., edited by S. Das, M. Ostrovsky, D. Pennock, and B. Szymanski. Berlin: Springer, lecture no ed.

Deluca, Anna and Álvaro Corral. 2013. "Fitting and goodness-of-fit test of non-truncated and truncated power-law distributions." Acta Geophysica 61 (6):1351-1394.

Dzhabarov, Constantine and William T. Ziemba. 2010. "Do seasonal anomalies still work?" The Journal of Portfolio Management 36 (3):93-104.

Fields, M.J. 1931. "Stock prices: A problem in verification." The Journal of Business of the University of Chicago 4 (4):415-418. 
French, Kenneth R. 1980. "Stock Returns and the Weekend Effect." Journal of Financial Economics 8:55-69.

Gabaix, Xavier, Parameswaran Gopikrishnanb, Vasiliki Plerou, and Eugene Stanley. 2007. "A unified econophysics explanation for the power-law exponents of stock market activity." Physica A: Statistical Mechanics and its Applications :81-88.

Gopikrishnan, Parameswaran, Vasiliki Plerou, Xavier Gabaix, and H Eugene Stanley. 2000. "Statistical Properties of Share Volume Traded in Financial Markets." Physical Review E 62:R4493-R4496.

Gultekin, M.N. and N.B. Gultekin. 1983. "Stock Market Seasonality: International Evidence." Journal of Financial Economics 12 (December):469-481.

Haugen, R.A. and Joseph Lakonishok. 1988. The Incredible January Effect. Homewood, IL: Dow-Jones-Irwin.

Jain, Prem and Gun-Ho Joh. 1988. "The Dependence between HoIGNOREy Prices and Trading Volume." The Journal of Financial and Quantitative Analysis 23 (3):269-283.

Lakonishok, Joseph and Edwin Maberly. 1990. "The Weekend Effect: Trading Patterns of Individual and Institutional Investors." The Journal of Finance 45 (1):231-243.

Malevergne, Y., V. Pisarenko, and D. Sornette. 2005. "Empirical distributions of stock returns: between the stretched exponential and the power law?" Quantitative Finance 5 (4):379-401.

Mantegna, Rosario and H Eugene Stanley. 2000. An introduction to econophysics. Cambridge University Press.

Mclean, R. David and Jeffrey Pontiff. 2016. "Does Academic Research Destroy Stock Return Predictability?" The Journal of Finance 71 (1):5-32.

Newman, M. E. J. 2004. "Power laws, Pareto distributions and Zipf's law." Contemporary Physics (46):323-351.

Officer, R.R. 1975. "Seasonality in Australian capital markets: Market efficiency and empirical issues." Journal of Financial Economics 2 (1):29-51.

Plerou, Vasiliki, Parameswaran Gopikrishnan, Xavier Gabaix, Luis A. Nunes Amaral, and H. Eugene Stanley. 2001. "Price fluctuations, market activity and trading volume." Quantitative Finance 1:2:262-269.

Plerou, Vasiliki, Parameswaran Gopikrishnan, Xavier Gabaix, and H Eugene Stanley. 2004. "On the Origin of Power-Law Fluctuations in Stock Prices." Quantitative Finance 4:C11-C15. 
Plott, CR and KY Chen. 2002. "Information aggregation mechanism: concept, design, and implementation for a sales forecasting problem."

Podobnik, Boris, Davor Horvatic, Alexander M Petersen, and H Eugene Stanley. 2009. "Cross-correlations between volume change and price change." PNAS 106 (52):22079-22084.

Poterba, J.M. and S.J. Weisbenner. 2001. "Capital gains tax rules, tax-loss trading, and turn-of-the-year returns." The Journal of Finance 51 (1):353368.

Praetz, PD. 1972. "The Distribution of Share Price Changes." Journal of business 45 (1):49-55.

Qiu, T., L.X. Zhong, G. Chen, and X.R. Wu. 2009. "Statistical properties of trading volume of Chinese stocks." Physica A: Statistical Mechanics and its Applications 388:2427-2434.

Reinganum. 1983. "The anomalous stock market behavior of small firms in January: Empirical tests for tax-loss selling effects." Journal of Financial Economics 12 (1):89-104.

Roll, Richard. 1983. "Vas ist das? The turn-of-the-year effect and the return premia of small firms." The Journal of Portfolio Management 9 (2):18-28.

Rozeff, M.S. and W.R. Kinney. 1976. "Capital Market Seasonality: the Case of Stock Returns." Journal of Financial Economics 3 (4):379-402.

Rubinstein, Mark. 2001. "Rational Markets: Yes or No? The Affirmative Case." Financial Analysts Journal 57 (3):15-29.

Sewell, Martin. 2011. "Characterization of financial time series." Research Note $R N / 11 / 01 \mathrm{UCL}$, London.

Sias, R.W. and L.T. Starks. 1997. "Institutions and individuals at the turn-ofthe-year." The Journal of Finance 52 (4):1543-1562.

Sornette, Didier. 2006. Critical phenomena in natural sciences: chaos, fractals, selforganization and disorder: concepts and tools. Berlin: Springer-Verlag, 2nd ed.

Thaler, Richard H. 1992. The Winner's Curse: Paradoxes and Anomalies of Economic Life. Princeton, New Jersey.

Wachtel, Syndey. 1942. "Certain observations on seasonal movements in stock prices." The Journal of Business of the University of Chicago 15 (2).

Wolfers, Justin and Eric Zitzewitz. 2006. "Prediction Markets in Theory and Practice." 